\title{
Augmentation of Clozapine with Aripiprazole in Severe Psychotic Bipolar and Schizoaffective Disorders: A Pilot Study
}

\author{
Alessandra Benedetti ${ }^{\S{ }^{*}}$, Antonello Di Paolo ${ }^{\wedge}$, Marianna Lastella^, Francesco Casamassima ${ }^{\S}$, Chiara \\ Candiracci ${ }^{\circ}$, Antonella Litta $^{\S}$, Laura Ciofi ${ }^{\wedge}$, Romano Danesi ${ }^{\wedge}$, Lorenzo Lattanzi ${ }^{\S}$, Mario Del Tacca ${ }^{\wedge}$ \\ and Giovanni Battista Cassano ${ }^{\S}$
}

\author{
§Department of Psychiatry, Neurobiology, Pharmacology and Biotechnologies, University of Pisa \\ ^Department of Internal Medicine, University of Pisa \\ - Psychiatric Clinic, Polytechnic University of Marche, Ancona \\ ${ }^{I}$ those Authors equally contributed to the study
}

\begin{abstract}
:
Aim: To evaluate the efficacy and safety of the augmentation of clozapine with aripiprazole in patients with treatmentresistant schizoaffective and psychotic bipolar disorders in a retrospective manner. Pharmacodynamic and pharmacokinetic interactions between the two drugs were also investigated.

Patients: Three men and 4 women (median age 36 and 40 years, respectively) who had mean scores at BPRS and CGISeverity of $59.1 \pm 12.0$ and $5.4 \pm 0.5$, respectively, were treated with clozapine (mean dose $292.9 \pm 220.7 \mathrm{mg} / \mathrm{day}$ ). Patients received an adjunctive treatment with aripiprazole (mean dose $6.8 \pm 3.7 \mathrm{mg} / \mathrm{day}$ ). Clozapine, norclozapine and aripiprazole plasma levels were measured by means of a high performance liquid chromatograpy with UV detection.

Results: Total scores at BPRS decreased significantly (from 59.1 \pm 12.0 to $51.1 \pm 15.6, \mathrm{p}=0.007$ ) after aripirazole augmentation. In particular, the factors "thought disorder" (from 10.4 \pm 4.4 to $9.0 \pm 4.5, \mathrm{p}=.047$ ) and "anergia" (from $10.0 \pm 2.7$ to $8.0 \pm 2.4, \mathrm{p}=.018$ ) significantly improved. Concomitant administration of aripiprazole and clozapine did not result in an increase in side effects over the period of treatment. Dose-normalized plasma levels of both clozapine and norclozapine and the clozapine/norclozapine metabolic ratio in all patients did not vary as well.

Conclusion: The augmentation of clozapine with aripirazole was safe and effective in severe psychotic schizoaffective and bipolar disorders which failed to respond to atypical antipsychotics. A possible pharmacokinetic interaction between clozapine and aripiprazole does not account for the improved clinical benefit obtained after aripiprazole augmentation.
\end{abstract}

Keywords: Psychotic bipolar disorder, schizoaffective disorder, clozapine, aripiprazole, augmentation, pharmacokinetic interactions.

\section{INTRODUCTION}

Severe forms of psychotic mood disorders may be characterized by psychotic manifestations typically schizophrenic (first-rank symptoms), or residual negative symptoms and deterioration of functioning $[1,2,3]$. Such clinical pictures, often labelled schizoaffective, represent intermediate syndromes between typical bipolar disorder and typical schizophrenia. They are frequently treatment-resistant to classical mood-stabilizers so that a maintenance treatment with antipsychotics is often considered [4]. Actually, these patients undergo complex treatment regimens, which may include the co-administration or more mood-stabilizer and one or more first- or second-generation antipsychotics. Clozapine, the "gold standard" of second-generation antipsy-

*Address correspondence to this author at the Department of Psychiatry, Neurobiology, Pharmacology and Biotechnologies, University of Pisa, Via Roma 57, 56126 Pisa - Italy; Tel: +39-050-835411; Fax: +39-050-21581; E-mail: a.benedetti@med.unipi.it, benedettia@hotmail.it chotics, has not been approved for the treatment of bipolar disorder, though it has showed its efficacy in acute mania [5] and in the prevention of recurrences of severe psychotic bipolar disorders [6]. Aripiprazole, which is a partial agonist at $\mathrm{D}_{2}$ and $5-\mathrm{HT}_{1 \mathrm{~A}}$ receptors while exerts antagonist activity against $5-\mathrm{HT}_{2 \mathrm{~A}}$ receptors, has been approved by FDA for the maintenance treatment after a manic or mixed episode.

The combination of clozapine and aripiprazole in treatment-resistant psychoses is effective and well-tolerated according to a recent review [7]. However, large studies are not yet available and the majority of the published researches are confounded by several methodologic flaws. A large, randomized, multicenter trial is ongoing in Italy to test the efficacy of clozapine - aripiprazole combination compared to clozapine - haloperidol combination [8].

Recently, a double-bind placebo controlled trial of aripiprazole augmentation of clozapine, reported no significant improvement of total symptom severity but a favorable 
change in the negative symptomatology in a sample of 62 patients with schizophrenia [9].

These data appear to be in line with case reports and open-label trials suggesting that this combined treatment may improve residual and negative symptoms in schizophrenia with no change in clozapine serum levels after administration of aripiprazole [10-16]. In fact, the two drugs have different metabolic pathways, therefore no relevant interactions are expected to occur, although other pharmacokinetic interactions could also be possible but not yet explored.

Since no studies have tested such a treatment strategy in schizoaffective or severe psychotic bipolar disorder, we aimed at evaluating in a retrospective fashion the efficacy and the safety of augmentation of clozapine with aripiprazole in those kind of patients, and to assess any possible pharmacokinetic interaction between the two drugs.

\section{MATERIALS AND METHODOLOGY}

\section{Patients}

Medical records of adult inpatients with a diagnosis of schizoaffective or psychotic bipolar disorders (according to DSM-IV criteria [17]) and treated with clozapine and aripiprazole were retrospectively and thoroughly examined referring to a six month period before enrolment in the study. All patients were hospitalized in the Wards of the Department of Psychiatry, at the University of Pisa, where a number of therapeutical monitoring protocols are active. These include the administration of rating scales, the recording of side effects, laboratory tests and measurement of drug levels.

Eligibility criteria were: 1) age within the range 18-65 years, 2) failure of at least two trials with different classical mood stabilizers, and/or with antipsychotics 3) administration of clozapine for at least six months at the most tolerated dosage, 4) persistent positive, negative or depressive symptoms, or relevant mood swings (despite the use of clozapine), 5) eligibility for aripiprazole augmentation and 6) registration of plasma levels of clozapine and aripiprazole beacause of the application of therapeutic drug monitoring protocols for both drugs. Further inclusion criteria were: 7) normal values of bone marrow, hepatic and renal functions, 8) capability to attend the follow up visits, 9) proved compliance. Exclusion criteria included: 1) positive case history of drug abuse, 2) Parkinson's disease, 3) epilepsy, 4) myasthenia gravis, 5) current major depression, 6) jaundice or hematological diseases, 7) photosensitization, 8) pregnancy or breast-feeding. Smoking habits were recorded for each patient, while the administration of other - antipsychotic, mood stabilizers, or antidepressant - drugs were allowed.

Clozapine and norclozapine plasma levels recorded within patients' case report forms were considered for the aims of the present work at the day before (T1) and 15 days after (T2) aripiprazole administration. On the contrary, aripiprazole plasma levels measured at least ten days after the first administration (T2) were considered suitable for the present study. In particular, the therapeutic monitoring protocols adopted in our clinic were based on the measurement of plasma drug levels three hours after clozapine and aripiprazole morning dose, which corresponds approximately to the time of peak concentration of both drugs, in fasting conditions.

ECG, safety hematology, including complete blood count and blood chemistry tests for liver function were performed on admission and during adjunctive treatment according to our routine protocols. Unwanted effects were assessed by evaluating patients at baseline and at T2. Seven patients were required in order to detect an increase of $\geq 25 \%$ in the mean values of clozapine's plasma concentration before and after augmentation with aripiprazole, with $80 \%$ power at a $5 \%$ level of statistical significance, and when a standard deviation of $20 \%$ of mean values was assumed.

All patients provided written consent to the revision of their case histories, and the study was authorized by the local Ethics Committee.

\section{Evaluation of the Disease, Treatment Efficacy and Tolerability}

Before the administration of aripiprazole (T1) and 15 days after the augmentation (T2), characteristics and severity of patients' disease, together with treatment effectiveness evaluated according to the Brief Psychiatric Rating Scale-24 items (BPRS; [18]) and Clinical Global Impression (CGI; [19]) scales were recorded. Furthermore, any side effect occurred during the study was evaluated in the Dosage Record and Treatment Emergent Symptom Scale (DOTES; [20]).

\section{Laboratory Analysis}

Therapeutic drug monitoring protocols provided that, blood samples $(5 \mathrm{ml})$ were withdrawn from a peripheral vein of the forearm, collected within heparinized tubes and immediately centrifuged. Plasma was stored at $-20{ }^{\circ} \mathrm{C}$ until the measurement of drug levels.

Plasma concentrations of clozapine and its active metabolite were determined by high performance liquid chromatography (HPLC) method by using a commercially available kit (Chromsystems, Munchen, Germany), following the instructions of manufacturers.

The same kit was used, without modifications, for the measurement of aripiprazole levels in plasma. In our laboratory, the method was proven to be linear for clozapine and norclozapine from $10 \mathrm{ng} / \mathrm{mL}$ (limit of quantitation) up to 1.5 $\mu \mathrm{g} / \mathrm{mL}$, which widely overlapped the therapeutic range of clozapine plasma levels. Furthermore, the method was linear from 10 up to $500 \mu \mathrm{g} / \mathrm{mL}$ in the case of aripiprazole, which could be considered as a temptative therapeutic range when the drug is administered at doses up to $30 \mathrm{mg} /$ day [21]. Finally, the method was characterized by an interday and intraday variability lower than $15 \%$ for all analytes.

The clozapine/norclozapine plasma concentration ratio was calculated as an index of drug metabolism in patients, while levels of the drug and the metabolite were normalized by the daily clozapine dose in order to reduce interpatient variability because of different daily dosages.

\section{Statistical Analysis}

Results are expressed as mean value \pm standard deviation and median. Paired Student's t test was performed by Intercooled Stata 8.2 [22] to evaluate differences between periods 
Table 1. Demographic, Clinical Characteristics and Treatments $(\mathrm{N}=7)$

\begin{tabular}{|c|c|c|c|c|c|c|c|c|c|}
\hline $\mathbf{P}$ & Sex & $\begin{array}{c}\text { Age } \\
\text { (Years) }\end{array}$ & Smoker & $\begin{array}{l}\text { Clozapine T1 } \\
\text { (mg/die) }\end{array}$ & $\begin{array}{l}\text { Clozapine } \\
\text { T2 (mg/die) }\end{array}$ & $\begin{array}{l}\text { Aripiprazole } \\
\text { (mg/die) }\end{array}$ & $\begin{array}{l}\text { \% of Im- } \\
\text { provement in } \\
\text { BPRS Total } \\
\text { Scores }\end{array}$ & $\begin{array}{c}\text { Scores at } \\
\text { CGI- I } \\
\text { (T2) }\end{array}$ & Concomitant Medications \\
\hline 1 & $\mathrm{~F}$ & 41 & no & 600 & 525 & 5 & 28.8 & 1 & HAL $10 \mathrm{mg}$, CDM $2.3 \mathrm{mg}$ \\
\hline 2 & M & 26 & yes & 200 & 200 & 7.5 & 22.5 & 1 & $\begin{array}{l}\text { HAL } 6.5 \mathrm{mg} \text {, LI } 600 \mathrm{mg} \text {, } \\
\text { VPA } 1000 \mathrm{mg} \text {, IMI } 50 \mathrm{mg}\end{array}$ \\
\hline 3 & $\mathrm{~F}$ & 56 & no & 150 & 100 & 5 & 15.1 & 3 & $\begin{array}{c}\text { Nortriptiline } 20 \mathrm{mg}, \mathrm{LI} 450 \\
\mathrm{mg}, \mathrm{CDM} 1.9 \mathrm{mg}\end{array}$ \\
\hline 4 & $\mathrm{~F}$ & 35 & yes & 600 & 600 & 15 & 0 & 4 & HAL $15 \mathrm{mg}$, CDM 7.5mg \\
\hline 5 & M & 42 & no & 100 & 25 & 5 & 10.9 & 3 & $\begin{array}{c}\text { HAL } 1 \mathrm{mg} \text {, IMI } 150 \mathrm{mg} \text {, } \\
\text { Duloxetine 60mg, CDM } 2 \\
\mathrm{mg}\end{array}$ \\
\hline 6 & M & 36 & yes & 300 & 300 & 5 & 9.4 & 3 & $\begin{array}{l}\text { HAL } 2 \mathrm{mg}, \text { LI } 900 \mathrm{mg}, \\
\text { CDM } 1.15 \mathrm{mg}\end{array}$ \\
\hline 7 & $\mathrm{~F}$ & 39 & yes & 150 & 137.5 & 5 & 3.3 & 3 & HAL $2 \mathrm{mg}$ \\
\hline
\end{tabular}

P: patient; HAL: Haloperidol; VPA: Sodium Valproate; LI: Lithium; IMI: Imipramine; CDM: Clordemethyldiazepam.

of treatment (T1 vs. T2) in clozapine and norclozapine plasma levels. A p-value lower than 0.05 was considered to be significant.

\section{RESULTS}

\section{Patients and Treatment Effectiveness}

The enrolment for the study was active from June, 2008 up to February 2009. During that period of time, 7 patients affected by schizoaffective $(\mathrm{N}=6)$ or severe psychotic bipolar disorders ( $\mathrm{N}=1$, patient $\mathrm{n}$. 3) could be included in the study according to the inclusion/exclusion criteria. The median age was 39 years (range 26-56), 3 patients were men (median age, 36 years) and 4 women (median age, 40 years). Before aripiprazole augmentation, mean scores at BPRS and CGISeverity were $59.1 \pm 12.0$ and $5.4 \pm 0.5$, respectively. Table 1 displays demographic features, doses of clozapine and aripiprazole, and of the other drugs, and measures of outcome (percentages of improvement at BPRS and scores at CGI-I) for each patient.

Before study initiation, patients had been assuming clozapine at a mean dosage of $292.9 \pm 220.7 \mathrm{mg} /$ day (range 100$600 \mathrm{mg} /$ day) for at least one year except for one patient who had been assuming clozapine for six months. All patients were at the steady state for clozapine and previous controls demonstrated very stable plasma concentrations.

Aripiprazole was added to the previous therapy in order to improve symptomatology with doses ranging from 5 to 15 $\mathrm{mg}$ /day (mean dose $6.8 \pm 3.7 \mathrm{mg} /$ day).

Concomitant medications did not vary in the study period, except for a man who shifted from paroxetine 10 $\mathrm{mg} /$ day to duloxetine $60 \mathrm{mg} /$ day.

After augmentation with aripiprazole, the mean daily dose of clozapine was 269.6 $\pm 218.4 \mathrm{mg}$ : particularly, in 4 patients the dose was reduced after 15 days because of the improvement of psychotic symtoms. In the remaining 3 subjects, the daily dose was unchanged (Table 1). All patients treated with haloperidol had been taking this drug for a 10 to 30 days period before study initiation.

The introduction of aripiprazole led to a significant improvement in BPRS total score $(59.1 \pm 12.0$ at T1 vs. $51.1 \pm 15.6$ at $\mathrm{T} 2$, respectively, $\mathrm{p}=0.007$ ). One patient (male, patient number 6) manifested an increase in motor activity and agitation four days after the augmentation with aripiprazole and we decided to discontinue the add-on treatment. Only six patients who remained in the study are included in the analyses.

In particular, the analyses of the 4 BPRS factor scores according to Ventura et al. [18] highlighted that the positive symptoms factor (including the items 9, 10 11, 12, 14 corresponding to suspiciousness, hallucinations, unusual thought content, bizzarre behavior, and disorientation) significantly improved ( $16 \pm 5.4$ at $\mathrm{T} 1$ vs $13.5 \pm 6.5$ at $\mathrm{T} 2, \mathrm{t}=2.7 . \mathrm{p}=0.4$ ), similarly to the negative symptoms factor (including the items 13, 1617 , and 18 corresponding to self-neglecting, blunted affect, emotional withdrawal, and motor retardation) $(10.5 \pm 3.3$ at $\mathrm{T} 1$ vs $7.8 \pm 2.1$ at $\mathrm{T} 2, \mathrm{t}=2.8, \mathrm{p}=0.4)$.

At the section improvement of CGI scale, four patients scored 3 (Minimally improved), one scored 4 (No change), and two patients scored 1 (very much improved). With these Regard, the higher improvement was in the BPRS positive and negative symptoms factors for the first patient (respectively $38 \%$ and $46 \%$ ) and in positive symptoms and in mania factors for the other (both factors an improvement of $31 \%$ ).

Among 4 patients who scored 3 at CGI-I, one had the higher improvement in BPRS depression-anxiety factor $(27 \%)$, another in positive and manic symptoms factor (respectively $15 \%$ and $14 \%$ ), two patients had the higher im- 
Table 2. Serum Concentrations (ng/m) and Ratio Concentration/Daily Dosages of Clozapine, Norclozapine, and Serum Concentrations (ng/m) of Aripiprazole at T1 and T2 (N=7)

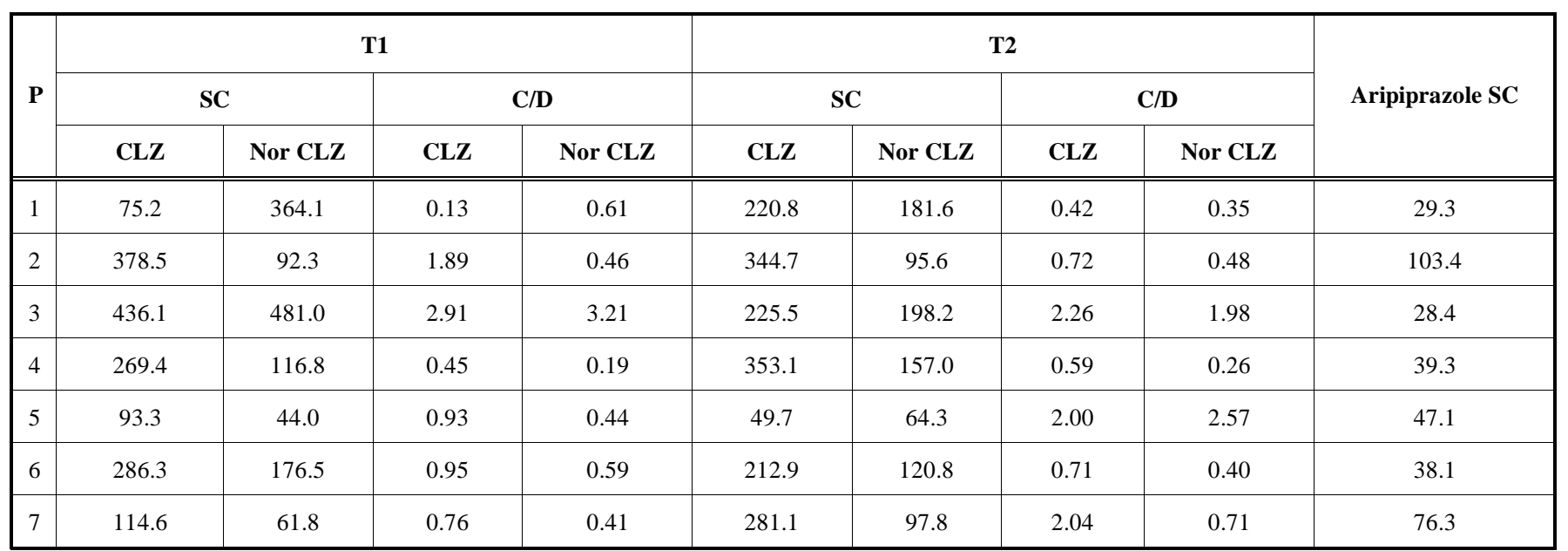

P: patient; SC : Serum Concentration; C/D: Ratio concentration/daily dosages; CLZ: clozapine; NorCLZ: norclozapine.

provement in negative symptomatology (respectively $40 \%$, and $31 \%)$.

Concomitant administration of aripiprazole and clozapine did not result in an increase in side effects over the period of treatment, whereas the appetite decreased in two patients.

\section{Plasma Levels of Drugs}

Table 2 displays serum levels of clozapine and norclozapine before and after augmentation with aripiprazole. The mean plasma concentrations of clozapine and norclozapine at T1 $(236.2 \pm 144.3 \mathrm{ng} / \mathrm{ml}$ and $190.9 \pm 167.2 \mathrm{ng} / \mathrm{ml}$, respectively) did not differ significantly from those measured at T2 $(241.1 \pm 102.4 \mathrm{ng} / \mathrm{ml}$ and $130.8 \pm 49.4 \mathrm{ng} / \mathrm{ml}$, respectively).

The same results were obtained after dose normalization of plasma concentrations of both clozapine and norclozapine $(1.1 \pm 1.0$ and $1.0 \pm 0.9$ at $\mathrm{T} 1$, and $1.2 \pm 0.8$ and $0.8 \pm 1.1$ respectively), which was introduced to reduce the interpatient variability because of the different dosages of clozapine.

Finally, the clozapine/norclozapine ratio at T2 $(1.95 \pm 1.00)$ remained unchanged in comparison with that calculated at T1 $(1.87 \pm 1.22)$, suggesting that an influence of aripiprazole on clozapine metabolism should be excluded.

In the remaining patients, aripiprazole plasma levels ranged from 28.4 up to $103.4 \mathrm{ng} / \mathrm{ml}$, achieving a mean value of $51.7 \pm 27.9 \mathrm{ng} / \mathrm{ml}$.

\section{DISCUSSION}

The present study suggests that the augmentation of clozapine with aripiprazole may allow a better control of at least some symptoms in the subjects suffering from schizoaffective and severe psychotic bipolar disorders, who failed to respond to the second-generation antipsychotic clozapine.

The pivotal aspect of the study was that, an augmentation strategy was evaluated in difficult-to-treat psychotic patients, for whom the benefit of treatment was relatively smaller than expected. Psychotic bipolar and schizoaffective patients resistant to mood stabilizers may need long-term treatment with atypical antipsychotics, including clozapine. Not with- standing the clinical efficacy of clozapine due to its serotoninergic and dopaminergic activity leads to significant clinical improvement [23], the drug does not provide a complete remission of positive or negative symptoms in a substantial percentage of patients $[24,25,26,6,27,28]$, and augmentation strategies are required.

After aripiprazole augmentation all patients improved significantly in BPRS total scores, and particularly in "positive symptoms" and "negative symptoms" factors. Two out of 7 patients $(28.57 \%)$ substantially ameliorated and 4 $(57.14 \%)$ patients slightly improved. Given the small sample size, including patients with a severe clinical course, these findings may be considered clinically promising. A larger sample size and a longer follow-up evaluation could allow to estabilish, whether improvements diminish, are maintained, or even increase over time.

A possible pharmacokinetic interaction between clozapine and aripiprazole did not account for the improved clinical benefit obtained because dose-normalized plasma levels of both clozapine and norclozapine did not change, and the clozapine/norclozapine metabolic ratio was unchanged in all patients. Therefore, the amelioration in BPRS and CGI scores could be due to the particular mechanism of action of aripiprazole [29]. In fact, the drug acts as a D2 partial agonist displaying a $30-40 \%$ activity of a full D2 agonist, while it is able to occupy more than $95 \%$ of D2 receptor [30]. Therefore, aripiprazole exerts a total inhibitory effect on nearly $65 \%$ of D2 receptors, but sparing the dopaminergic projections from a deep inhibitory control, despite its high affinity against these receptors [31, 29]. These characteristics of aripiprazole lead to a controlled and ameliorated firing in a hypodopaminergic environment created by the clozapineinduced reduction of dopamine hyperactivity. This pharmacodynamic interaction may result in the decrease in symptoms, such as emotional withdrawal, motor retardation, blunted affect and disorientation [10].

The majority of studies did not report any specific side effects with the combination of clozapine and aripiprazole. 
However, two cases of dyskinesia and malignant neuroleptic syndrome were reported in a sample of 26 patients [32].

In our sample, the augmentation of clozapine with aripiprazole resulted safe. In fact, no side effects occurred after the augmentation with aripiprazole as assessed with DOTES Scale, if a reduction of appetite in two patients is excluded. Particularly, patients underwent a close EKG monitoring because of the potential cardiotoxicity of the antipsychotic association, but no cardiologic adverse events were apparent. However, one patient discontinued the treatment with aripiprazole because motor agitation occurred. It was not clear, whether this was due to the natural course of illness or to the combined action of aripiprazole administration and the reduction of clozapine dosage. However, psychomotor activation in this case was limited to a short time frame and did not influence negatively illness course.

Moreover, in our study, which enrolled patients with a diagnosis of affective disorders, apparently at higher risk for treatment-emergent extrapyramidal symptoms (EPS) as compared to patients with schizophrenia [33], no extrapyramidal side effects were observed during the combination treatment. Finally, in the literature, there is a growing body of evidence concerning the increased risk of cardiac toxicity and the use of older and newest antipsychotics, as well as clozapine [34]. Because of that issue, our patients underwent a careful cardiac evaluation before treatment and during follow up visits, but none of them experienced signs or symptoms of cardiac toxicity (i.e., arrhythmias). Therefore in the present study, that kind of adverse reactions was not observed, but it is still strongly recommended a close monitoring of patient's health conditions.

\section{CONCLUSIONS}

In conclusion, despite substantial limitations represented by the retrospective design, the small sample size, and the short period of follow-up, results from the present study suggest that the augmentation of clozapine with aripirazole may be safe and effective in schizoaffective or severe psychotic bipolar disorders, which failed to respond to clozapine.

\section{CONFLICT OF INTEREST}

All authors declare that there are no financial interests or conflicts of interest to disclose.

\section{NOTE}

Study protocol was approved by the Ethical Commitee of University of Pisa on January $17^{\text {th }}, 2008$ (registration number 2449). Because the study was retrospective, the registration within the national registry was not required, and an EUDRACT code was not given.

\section{REFERENCES}

[1] Goodwin FK, Jamison KR, Eds. Manic-depressive illness. New York: Oxford University Press 1990.

[2] Goldberg JF, Harron M, Eds. Bipolar disorders: clinical course and outcome. Washington, DC: American Psychiatric Press 1999.

[3] Judd LL, Akiskal HS. The prevalence and disability of bipolar spectrum disorders in the U.S. population: re-analysis of the ECA database taking into account subthreshold cases. J Affect Disord 2003; 73: 123-31.
[4] American Psychiatric Association. Practice guideline for the treatment of patients with bipolar disorder (revision). Am J Psych 2002;159 (suppl 4): 1-50.

[5] Calabrese JR, Kimmel SE, Woyshville MJ, et al. Clozapine for treatment-refractory mania. Am J Psychiatry 1996; 153(6): 759-64.

[6] Ciapparelli A, Dell'Osso L, Bandettini di Poggio A, et al. Clozapine in treatment-resistant patients with schizophrenia, schizoaffective disorder, or psychotic bipolar disorder: a naturalistic 48-month follow-up study. J Clin Psychiatry 2003; 64(4): 451-8.

[7] Englisch S, Zink M. Combined antipsychotic treatment involving clozapine and aripiprazole. Prog Neuropsychopharmacol Biol Psychiatry $2008 ; 1 ; 32(6): 1386-92$.

[8] Nosè M, Accordini S, Artioli P, et al. Rationale and design of an independent randomised controlled trial evaluating the effectiveness of aripiprazole or haloperidol in combination with clozapine for treatment-resistant schizophrenia. Trials $2009 ; 15 ; 10: 31$.

[9] Chang JS, Ahn YM, Park HJ, et al. Aripiprazole augmentation in clozapine-treated patients with refractory schizophrenia: an 8week, randomized, double-blind, placebo-controlled trial. J Clin Psychiatry 2008; 69(5): 720-31.

[10] Clarke LA, Lindenmayer JP, Kaushik S. Clozapine augmentation with aripiprazole for negative symptoms. J Clin Psychiatry 2006; 67, 4: 675-6.

[11] Abu-Tair F, Kopitz J, Bergemann N. Clozapine Augmented With Aripiprazole in 5 Patients With Schizophrenia. J Clin Psychopharmacol 2006; 26(6): 669-71.

[12] Rocha FL, Hara C.Benefits of combining aripiprazole to clozapine: three case reports. Prog Neuropsychopharmacol Biol Psychiatry 2006; 30(6): 1167-9.

[13] Henderson DC, Kunkel L, Nguyen DD, et al. An exploratory openlabel trial of aripiprazole as an adjuvant to clozapine therapy in chronic schizophrenia. Acta Psychiatr Scand 2006; 113(2): 142-7.

[14] Ashton KA. Indexing of reports on aripiprazole augmentation of clozapine. J Clin Psychiatry 2007; 68(2): 334-5.

[15] Ziegenbein M, Sieberer M, Calliess IT, Kropp S. Combination of clozapine and aripiprazole: a promising approach in treatmentresistant schizophrenia. Aust N Z J Psychiatry 2005; 39(9): 840-1.

[16] Mitsonis CI, Dimopoulos NP, Mitropoulos PA, et al. Aripiprazole augmentation in the management of residual symptoms in clozapine-treated outpatients with chronic schizophrenia: An open-label pilot study. Prog Neuropsychopharmacol Biol Psychiatry 2007; 30; 31(2): 373-7.

[17] American Psychiatric Association. Diagnostic and Statistical Manual of Mental Disorders, 4th edition. Washington DC: American Psychiatric Association 1994.

[18] Ventura J, Nuechterlein KH, Subotnik KL, Gutkind D, Gilbert EA Symptom dimensions in recent-onset schizophrenia and mania: a principal components analysis of the 24-item Brief Psychiatric Rating Scale. Psychiatry Research 2000; 97: 129-135.

[19] Guy W, Bonato RR, Eds. Manual for the ECDEU Assessment Battery.2. Rev ed. Chevy Chase, Md: National Institute of Mental Health; 1970: 12-1-12-6

[20] Guy W. Dosage Record and Treatment Emergent Symptoms scale (DOTES). ECDEU assessment manual for psychopharmacologyrevised. DHEW Publication No. ADM 76-338 1976: 223-244 US Department of Health, Education, and Welfare, Public Health Service, Alcohol, Drug Abuse, and Mental Health Administration, NIMH Psychopharmacology Research Branch, Division of Extramural Research Programs Rockville, MD, 1976.

[21] Kirschbaum KM, Muller MJ, Zernig G, et al. Therapeutic monitoring of aripiprazole by HPLC with column-switching and spectrophotometric detection. Clin Chem 2005; 51(9): 1718-21.

[22] StataCorp, College Station, TX, USA 2004.

[23] Hirose T, Kikuchi T. Aripiprazole, a novel antipsychotic agent: dopamine D2 receptor partial agonist. J Med Invest 2005; 52: 28490.

[24] Perry PJ, Miller DD, Arndt SV, Cadoret RJ. Clozapine and norclozapine plasma concentrations and clinical response of treatmentrefractory schizophrenic patients. Am J Psychiatry 1991; 148(2): 231-5.

[25] Zito JM, Volavka J, Craig TJ, Czobor P, Banks S, Vitrai J. Pharmacoepidemiology of clozapine in 202 inpatients with schizophrenia. Ann Pharmacother 1993; 27(10): 1262-9 
[26] Potkin SG, Bera R, Gulasekaram B, et al. Plasma clozapine concentrations predict clinical response in treatment-resistant schizophrenia. J Clin Psychiatry (1994); 55 (Suppl B): 133-6.

[27] Remington G, Saha A, Chong SA, Shammi C. Augmentation strategies in clozapine-resistant schizophrenia. CNS Drugs 2005; 19(10): 843-72.

[28] Advokat C. Differential effects of clozapine versus other antipsychotics on clinical outcome and dopamine release in the brain. Essent Psychopharmacol 2005; 6(2): 73-90.

[29] Hamamura T, Harada T. Unique pharmacological profile of aripiprazole as the phasic component buster. Psychopharmacology (Berl) 2007; 191(3): 741-3.

[30] Grunder G, Carlsson A, Wong DF. Mechanism of new antipsychotic medications: occupancy is not just antagonism. Arch Gen Psychiatry 2003; 60: 974-7.
[31] Kapur S, Seeman P. Does fast dissociation from the dopamine d(2) receptor explain the action of atypical antipsychotics? A new hypothesis. Am J Psychiatry 2001; 158: 360-9.

[32] Karunakaran K, Tungaraza TE, Harborne GC. Is clozapinearipiprazole combination a useful regime in the management of treatment-resistant schizophrenia? J Psychopharmacol 2007; 21(4): 453-6.

[33] Gao K, Kemp DE, Ganocy SJ, Gajwani P, Xia G, Calabrese JR.Antipsychotic-induced extrapyramidal side effects in bipolar disorder and schizophrenia: a systematic review. J Clin Psychopharmacol 2008; 28(2): 203-9.

[34] Pacher P, Kecskemeti V. Cardiovascular Side Effects of New Antidepressants and Antipsychotics: New Drugs, old Concerns? Curr Pharm Des 2004; 10(20): 2463-2475.

(C) Benedetti et al.; Licensee Bentham Open.

This is an open access article licensed under the terms of the Creative Commons Attribution Non-Commercial License (http://creativecommons.org/licenses/by-nc/3.0/) which permits unrestricted, non-commercial use, distribution and reproduction in any medium, provided the work is properly cited. 